\title{
Speciation of Coagulase Negative Staphylococcal Isolates from Clinically Significant Specimens and their Antibiogram
}

\author{
Mariraj Jeer, Jyoti R. Hundekar* and S. Krishna \\ Department of Microbiology, Vijayanagara Institute of Medical sciences, Bellary, India \\ *Corresponding author
}

\section{Keywords \\ Specimens, Antibiogram, Staphylococcal isolates and Pathogens \\ Article Info \\ Accepted: \\ 23 May 2017 \\ Available Online: \\ 10 June 2017}

\section{A B S T R A C T}

Coagulase Negative Staphylococci (CONS) are the indigenous flora of the human skin and mucous membrane. They are usually contaminants, when isolated from clinical specimens. CONS have become important nosocomial pathogens, accounting for $9 \%$ of all nosocomial infections. These infections are difficult to treat because of the risk factors and the multiple drug resistance of these organisms. A total of 74 clinically significant CONS isolated from pus, urine, blood, sputum, ear swabs. These isolates were initially identified by colony morphology, Gram staining, catalase test, slide coagulase test, tube coagulase test. After confirming the isolates as CONS, species-level identification was performed by simple, non-expensive conventional methods and antibiotic sensitivity testing was also carried out by Kirby-Buer's disc diffusion method. Among the 74 isolates, S. epidermidis was the most common species isolated, seen in $29.7 \%$, followed by $S$. hemolyticus (20.2\%), S. saprophyticus (14.8\%), S. lugdunensis (13.5\%), S. capitis (10.8\%), S. cohni (4\%), S. schleiferi $(2.7 \%)$, S. xylosus and S. hominis (1.3\% each), Most of the isolates showed resistance to penicillin (83.7\%) fallowed by Ampicillin (77\%), Erythromycin (54\%), Cotrimoxazole $(27 \%)$ gentamicin $(16 \%)$, amikacin $(12 \%)$ piperacillin/tazobactum and linezolide (3\% each). The increased recognition of pathogenic potential in CONS and emergence of drug resistance among them demonstrates the need to adopt simple laboratory methods to identify the species and determine the antibiotic resistant patterns. It will help the clinicians in treating the infections caused by CONS.

\section{Introduction}

Coagulase Negative Staphylococci (CONS) are the normal skin flora, have emerged as predominant pathogens in hospital acquired infections (Usha et al., 2013), often associated with implanted devices, such as joint prosthesis, shunts and intravascular catheters especially in very young, old, and immune compromised patients. These infections are difficult to treat because of the risk factors and the multiple drug resistant nature of the organisms. Hence study was undertaken to identify and speciate CONS and their antibiogram (Sarojgolia et al., 2015). The main objectives of this study include, to speciate CONS isolates from various clinical samples. To determine antibiotic susceptibility pattern of CONS by Kirby Bauer disc diffusion method.

\section{Materials and Methods}

The study was carried out in department of Microbiology, VIMS ballari for a period of 6 months from January 2016 to June 2016. 
A total of 74 clinically significant CONS isolated from pus, urine, blood, sputum and ear swabs. The isolates were identified as CONS by colony morphology, Gram stain, catalase test and coagulase test (slide and tube coagulase). Bacitracin susceptibility was performed to exclude Micrococci and Stomatococcus species (Singh et al., 2008).

After confirming the isolates as CONS, species-level identification was performed by simple, non-expensive conventional methods These include the ornithine decarboxylase test, nitrate reduction test, Voges-Proskauer test, urease test and fermentation of sucrose, lactose, maltose, mannose, mannitol, xylose and trehalose. Susceptibility to novobiocin and polymyxin B was performed as per the standard procedure (Washington et al.; Patricia; Collee et al.,) and antibiotic sensitivity testing was also carried out by Kirby- Bauer's disc diffusion method according to CLSI guidelines (2016).

Identification of CONS by simple scheme (Washington et al.; Patricia; Collee et al.,).

\section{Results and Discussion}

Among the 74 isolates, $S$. epidermidis was the most common species isolated, seen in $29.7 \%$, followed by $S$. hemolyticus (20.2\%), $S$. saprophyticus $(14.8 \%), \quad$ S. lugdunensis (13.5\%), S. capitis (10.8\%), S. cohni (4\%), S. schleiferi (2.7\%), S. xylosus and S. hominis (1.3\% each).

Most of the isolates showed resistance to penicillin $(83.7 \%)$ fallowed by Ampicillin (77\%), Erythromycin (54\%), Cotrimoxazole (27\%) gentamicin (16\%), amikacin (12\%) piperacillin/tazobactum and linezolide (3\% each).

Although the pathogenic role of CONS is now well established, the clinical significance of the various species is still being defined. We should not disregard any of the organisms until their clinical significance is resolved. In the hospital microbiology laboratory, nonaureus isolates are simply reported as CONS without speciation. Because there is increasing pathogenicity of these organisms, CONS should be identified to the species level by simple, reliable and preferably inexpensive methods (Usha et al., 2013).

In our study among 74 isolates of CONS, $S$. epidermidis is the most frequent isolate (29.7\%), followed by S. hemolyticus $(20.2 \%)$, S. saprophyticus (14.8\%) S. lugdunensis (13.5\%), S. capitis (10.8\%), S. cohni (4\%), S. schleiferi $(2.7 \%)$, S. xylosus and S. hominis (1.3\% each), this study correlates with various other studies, In our study, most commonly isolated species is S. epidermidis (29.7\%) which is similar to other studies as shown by Usha et al., (2013) (32\%), Golia et al., (2015) (46.3\%) and Rajyalakshmi Gunti et al., (2016) (42\%). The next common species in our study was $S$. hemolyticus $(20.2 \%)$ similar results were seen in Usha et al., (2013) (18\%) and Rajyalakshmi Gunti et al., (2016) (28\%). But in other studies, it was S. saprophyticus that was the second most common species.

In present study the CONS infection was more common in males $(48 \%)$ than females (36\%), which is similar to other studies as well, as males (59\%) and females (41\%) as shown by Usha et al., (2013) and males $(64.9 \%)$, females $(47 \%)$ shown by Golia et al., (2015). When the different age groups were compared most common age group affected was between 30 and 50 years.

In this study, out of 74 isolates of CONS, 29 $(39 \%)$ isolates from pus, $22(29.7 \%)$ isolates from urine samples, 18 (24.3\%) from sputum samples, 9 (12\%) from blood samples, (6\%) etc. 

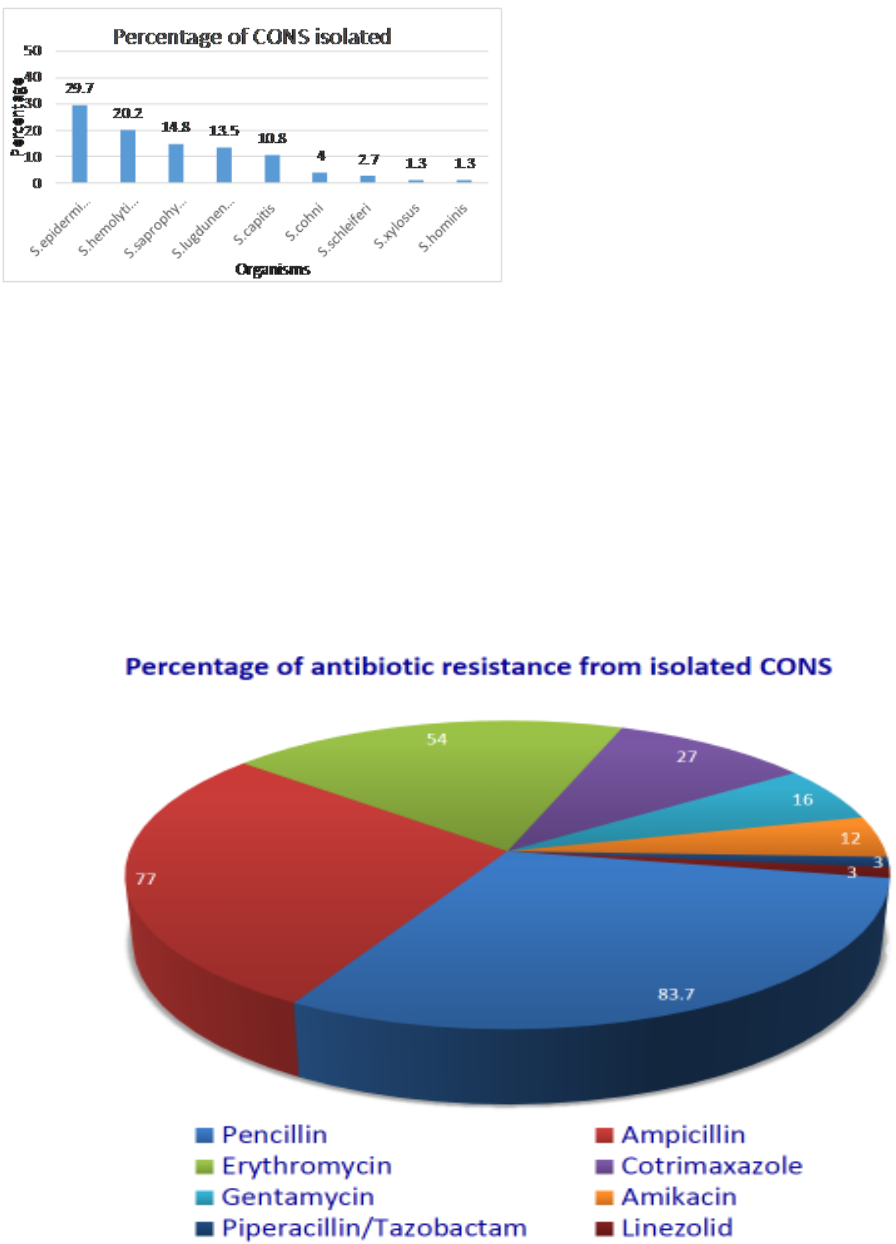

Our study reported out of $22 \mathrm{~S}$. epidermidis $8(36.3 \%)$ were isolated from sputum samples fallowed by $6(27.2 \%)$ from urine and $5(22.7 \%)$ from pus samples, but in a study by Shubhrasingh, Gopa Banaerjee et al., out of 60 S. epidermidis isolates, 30(50\%), were isolated from blood samples by $12(20 \%)$ from pus samples (Singh et al., 2008).

In the present study, most of CONS showed resistance to penicillin (83.7\%), followed by Ampicillin (77\%), Erythromycin (54\%), Cotrimoxazole (27\%) gentamicin (16\%), amikacin (12\%) piperacillin/tazobactum and linezolide (3\% each), no resistance to Vancomycin was seen in this study, most of CONS showed resistance to pencillin (83.7\%),which is correlating with the studies by Golia et al., (2015) (95.5\%) and Rajyalakshmi Gunti et al., (2016) (90\%). As well our study correlating with a study by Shubhrasingh, Gopa Banaerjee et al., where antibiotic susceptibility testing showed maximum resistance to penicillin and ampilicillin80\% and 38\% strains showed resistance to oxacillin (Singh et al., 2008). CONS, primarily $S$. epidermidis and $S$. hemolyticus are often resistant to multiple antibiotics and glycopeptides have been considered the drugs of choice for management of infections caused by these organisms (Silvia et al., 1992) and in a study by Del's Alamo, Cereda et al., (1999) showed that glycopeptide resistance is emerging among CONS isolate.

CONS have become the major cause of nosocomial blood stream infections as a result of combination of increased use of intravascular devices and an increase number 
of hospitalised immune compromised patients. S. epidermidis and S. hemolyticus are the common isolates identified and CONS are often resistant to multiple antibiotics (penicillin, ampicillin, oxacillin and etc.) and glycopeptides have been considered as the drugs of choice for management of infections caused by these organisms.

The increased pathogenic potential and multiple drug resistance demonstrate the need to adopt simple, reliable and non-expensive methods to identify the species and determine the antibiotic resistant patterns. It will help the clinicians in treating the infections caused by CONS.

\section{References}

CLSI guideline. 2016.

Collee, J.G., A.G. Fraser, B.P. Marmion, A. Simmons. Mackie and McCartney Practical medical microbiology: 14 ${ }^{\text {th }}$ edi: 247.

Del' Alamo, L., Cereda, R.F., Tosin, I. 1999. Antimicrobial susceptibility of coagulase negative Staphylococci and characterization of isolates with reduced susceptibility to glycopeptides. Diagn. Microbiol. Infect. Dis., 34(3): 185-191.

Goyal, R., Singh, N.P., Kumar, A., Kaur, I., Singh, M., Sunita, N., et al. 2006. Simple and economical method for speciation and resistotyping of clinically significant coagulase negative Staphylococci. Indian J. Med. Microbiol., 24: 201-4.

Patricia, M. Tille: Bailey and Scott's Diagnostic Microbiology: $13^{\text {th }}$ edi., 238-242.

Rajyalakshmi Gunti, Durgarani Arava, Rajashekhar Koppada. 2016. Speciation of coagulase negative Staphylococci and their abtibiogram: IOSR J. Dent. Med. Sci., 15(1): pp-28-31.

Sarojgolia, Deepali Bhimacharya Telsang, et al. 2015. Speciation of clinically significant coagulase negative Staphylococci and their antibiotic resistant patterns in tertiary care hospital: Int. J. Res. Med. Sci., 3(5): 12421246.

Silvia Natoli, Carla Fontana, et al. 1992. Characterization of coagulase-negative staphylococcal isolates from blood with reduced susceptibility to glycopeptides and therapeutic options J. Antimicrob. Chemother., 29: 459-466.

Singh, S., Banerjee, G., Agarwal, S.K., Kumar, M., Singh, R.K. 2008. Simple method for speciation of clinically significant Coagulase Negative Staphylococci and its antibiotic sensitivity/resistant pattern in NICU of tertiary care centre. Biomed. Res., 19: $97-101$

Singh, S., Banerjee, G., Agarwal, S.K., Kumar, M., Singh, R.K. 2008. Simple method for speciation of clinically significant Coagulase Negative Staphylococci and its antibiotic sensitivity/resistant pattern in NICU of tertiary care centre. Biomed. Res., 19: 97-101.

Usha, M.G., Shwetha, D.C., Vishwanath, G. 2013. Speciation of coagulase negative staphylococcal isolates from clinically significant specimens and their antibiogram: Indian J. Pathol. Microbiol., 56(3): 258-260.

Washington Winn, Jr., Stephen Allen, William, J. and Elmer Koneman. Gary Procop, Paul Schreckenberger, Gall Woods: Koneman's Color Atlas and Textbook of Diagnostic Microbiology: $6^{\text {th }}$ edi., 650-652.

\section{How to cite this article:}

Mariraj Jeer, Jyoti R. Hundekar and Krishna, S. 2017. Speciation of Coagulase Negative Staphylococcal Isolates from Clinically Significant Specimens and their Antibiogram. Int.J.Curr.Microbiol.App.Sci. 6(6): 1824-1827. doi: https://doi.org/10.20546/ijcmas.2017.606.212 